\title{
MiR-520c and MiR-519d Function as Oncogenes in Esophageal Cancer
}

NG Dasjerdy ${ }^{1}$, MS Javad ${ }^{2}$, G Masoumeh $^{1}$, A Shahryar ${ }^{3}$, M Samaie Nader ${ }^{1}$

\begin{abstract}
Objectives: Esophageal cancer is a poorly characterized deadly cancer with a malignancy ratio of $2 / 3$ among Iranian males and females, respectively. Recent studies on miR-520c and miR-519d have evidenced their either oncogenic or tumor suppressing roles in different cancers. The goal of this study was to assay the altered expressions of miR-520c and miR-519d in esophageal tumor tissues and adjacent non-tumor tissues in patients suffering from esophageal cancer.

Methods: RNA was extracted from 36-pair paraffin-embedded tumor and adjacent non-tumor tissues using TRIZOL kit and relative expressions of miR-520c and miR-519d were quantified by Real Time and RT-PCR using specific predesigned primers. CT method $\left(2^{-\Delta \Delta C T}\right)$ and SPSS16 were used for statistical calculations.
\end{abstract}

Results: Statistical analysis has revealed spectacular upward expression increases of 10.4 and 24.5 folds of miR-520c and miR-519d adjacent in Esophageal Squamous Cells compared to their non-tumor tissues $(\mathrm{P}<0.05)$.

Conclusion: In general, the present study being the first report on the evaluation of miR-520c and miR519d expressions in ESCC, is able to highlight the oncogenic roles of the two miRNA in ESCC and introduce them as appropriate potential alternatives to be utilized for further research on clinical treatment.

Keywords: Oncogene, MiR-520c, MiR-519d, Esophageal cancer

From: ${ }^{1}$ Department of Human genetics, Faculty of Advanced Technology, Golestan University of Medical Sciences, Gorgan, Iran. ${ }^{2}$ Department of Molecular Genetics, Faculty of Biological Sciences, Tarbiat Modares University, Tehran, Iran. ${ }^{3}$ Department of Pathology, Hakim Jourjani Hospital, Gorgan, Iran.

Correspondence: Dr M Samaie, Department of Human Genetics, Faculty of Advanced Medical Technology, Golestan University of Medical Sciences, Gorgan, Iran. Fax: 00981732430564, e-mail: n_samaei@yahoo.com 


\section{INTRODUCTION}

Cancer has remained one of the most common leading causes of death worldwide. As estimated, there will have been an increase of $45 \%$ in various types of cancer in developing countries by 2030 (1). Esophageal cancer (EC) is the sixth leading fatal cancer around the world (2). Squamous cell carcinoma (ESCC) and Adenocarcinoma (ADC) are two distinctive histological

subtypes of EC (3). In this respect, Golestan Province, in Iran, is one of the regions with highly prevalent incidences of EC with over $90 \%$ of ESCC, worldover (1).

Despite surgery and advances in the treatment of EC, the prognosis and survival rates for both types of this cancer are still dismal(4). Otherwise stated, Proportional to the time of cancer detection, life expectancy for the next five years will be less than $15 \%$ in patients with advanced tumor compared to $90 \%$ for those diagnosed at the first stage and underwent surgery $(3,5)$. Therefore, as conventional diagnostic strategies are not able to detect ESCC in the earliest stages, there is an urgent request for novel biomarkers which are less invasive and more powerful, and are capable of detecting ESCC at early stages $(3,4)$.

Recent innovations in miRNAs profiling technology have supported its great potentials in cancer diagnosis and treatment monitoring $(6,7)$. Small non-coding RNA molecules known as miRNA are single-stranded, highly conserved RNAs with approximate nucleotide length of 22 , which are able to modulate the stability, translation efficiency and regulation of target gene expression through binding to the 3 'un-translated regions (UTR) of their messenger RNAs $(8,9)$. Due to perfect or imperfect pairing of miRNA with target site in the massager RNA, these miRNAs will be able to regulate hundreds of individual mRNAs (10-12).

Although the gene networks and molecular mechanisms orchestrated by miR-519d and miR-520c are not completely recognized, expressive alterations of these two miRNA have been 
characterized in several cancers $(13,14)$. According to these studies, in hepatocellular carcinoma and prostate cancer, miR-519d performs its roles by targeting mki67 and p21, both of which are crucially important in cell cycle, tumorigenesis, and development and survival rate of EC $(2,8,9,15-18)$. Expressive alterations of miR-520c have been reported in different cancers including thyroid adenoma, liver, breast and fibroblast cancer, as well. So far, none of these two miRNAs has been investigated in EC $(5,6,14,19-24)$. The goal of this study was to assay the expressive alterations of miR-519d and miR-520c in esophageal tumor and adjacent non-tumor tissues, in order to discover putative novel biomarkers for detecting, screening and surveillance of EC.

\section{MATERIALS AND METHODS}

Tissue collection and processing

Thirty-six pairs of formalin-fixed paraffin-embedded (FFPE) esophagus tissues, consisting human ESCC and normal adjacent tissues from the same patients were obtained from the archives of Jorjani and Shariati Hospitals (Iran). Moreover, the patients' age and gender information were gathered from those Hospitals. H-E stained slides were reviewed by an experienced pathologist to identify the tumoral ESCC regions as well as its corresponding adjacent normal specimens for the cores to be carefully cut from FFPE blocks and placed in RNase free $2.0 \mathrm{ml}$ micro tubes for RNA isolation. This study was approved by the Golestan University of Medical Sciences Ethical Board.

\section{RNA isolation from FFPE specimens}

RNA was extracted from 72 samples by tissue weight of about $20 \mathrm{mgr}$. Initially, samples were trimmed of exceeding wax and deparaffined by three repeated rinses of $1 \mathrm{ml} 100 \%$ xylen for 10 - 
second vortex, followed by 3 -minute centrifugation at $13000 \mathrm{xg}$ in $22^{\circ} \mathrm{C}$. The same steps were repeated three times for $100 \%$ ethanol. The pellet was also allowed to air dry in room temperature.

The samples were sub-merged in $200 \mu \mathrm{l} 1 \mathrm{x}$ protease K digestion buffer containing $10 \mu$ of 20mg/ml protease $\mathrm{K}$ solution ( Fermentase, Lithuania) and $190 \mu \mathrm{l} \mathrm{PK}$ buffer (1 mM EDTA, $1 \mathrm{Mm} \mathrm{NaCl}, 5 \mathrm{Mm}$ Tris-Hcl, $\mathrm{PH}=8$ ), followed by the incubation of $4 \mathrm{hr}$ at $56^{\circ} \mathrm{C}$. Trizol solution (Invitrogen, USA) was then added to each sample tube, and purification was performed according to the manufacturing company's instructions. The quantified analysis of resulted RNA was performed by Pico drop spectrophotometer and OD 260/280 nm ratio.

Reverse Transcription and Quantitative Real-Time PCR

Using PARSGENOME miR-AMP kit (Iran) 1-2 ng of total RNA was reversely transcribed and amplified according to the manufacturer's protocol. Specific poly-A primers designed by PARSGENOME miR-AMP company were also used for the detection of miRNAs by quantitative Real-Time PCR using SYBR Green. The method is based on three following steps. Firstly, the length of miRNAs was extended with Poly-A enzyme. Secondly, the firststrand cDNA was synthesized with specific designed primers and, finally, it was amplified with ABI-7300 Real-Time PCR system (Applied Biosystem, USA).

The evaluations of miR-519d and miR-520c, target genes, expressions and miR-16 as endogenous were performed using SYBER Green. In this method, to perform Real-Time quantitative PCR, thermal cycling comprised with an initial incubation of $95^{\circ} \mathrm{C}$ for 3 min, which was followed by 40 cycles of denaturation at $95^{\circ} \mathrm{C}$ for 5 seconds. It was preceded by annealing at $63^{\circ} \mathrm{C}$ for 15 seconds and proliferation at $72^{\circ} \mathrm{C}$ for 30 seconds. 
PCR was prepared in a final volume of $20 \mu$ and each measurement was performed in triplets. Real-Time PCR electrophoresis was performed on 3\% agarose gel for further validation. To compare each miRNA expression among different specimen groups (tumor/non-tumor), normalization was performed based on miR-16 gene expression. Relative expression of target miRNAs was calculated by $2^{-\Delta \Delta \mathrm{Ct}},-\Delta \Delta \mathrm{Ct}=-(\Delta \mathrm{Ct}$ sample $-\Delta \mathrm{Ct}$ control $)(25)$.

\section{Calculation}

To compare the groups, T-test was used. For further calculations, CT method and SPSS 16 were used, and $\mathrm{P}$ values of $0<0.05$ were considered statistically significant. Pearson coefficient was performed to analyze the correlation of gene expressions.

\section{RESULTS}

In this study, the evaluation of miR-519d and miR-520c expressions was performed in 36 pairs of tumor/non tumor ESCC tissues by Real-Time PCR. Baseline clinical characteristics patient samples are summarized in Table 1. 
Table 1: Baseline clinical characteristics of patients' samples

\begin{tabular}{lcc}
\hline Parameter & Frequency & Percent (\%) \\
\hline Sex & 19 & 52.7 \\
Male & 17 & 47.2 \\
Female & & \\
Age ( Age range : 39_84, Median age: 61) & 25 & 69.4 \\
$>60$ & 11 & 30.6 \\
$<60$ & & \\
Differentiation & 30 & 83 \\
Well differentiation (low grade) & 6 & 17 \\
Poor differentiation (high grade) & & \\
\hline
\end{tabular}

Statistical analysis revealed significant expression rises of 10.4 and 24.5 folds of miR-519d and miR-520c in esophageal squamous cells compared to their adjacent non-tumor tissues, $95 \%$ confidence interval and $\mathrm{P}$ value $<0.05$ were considered statistically credited (fig1). 


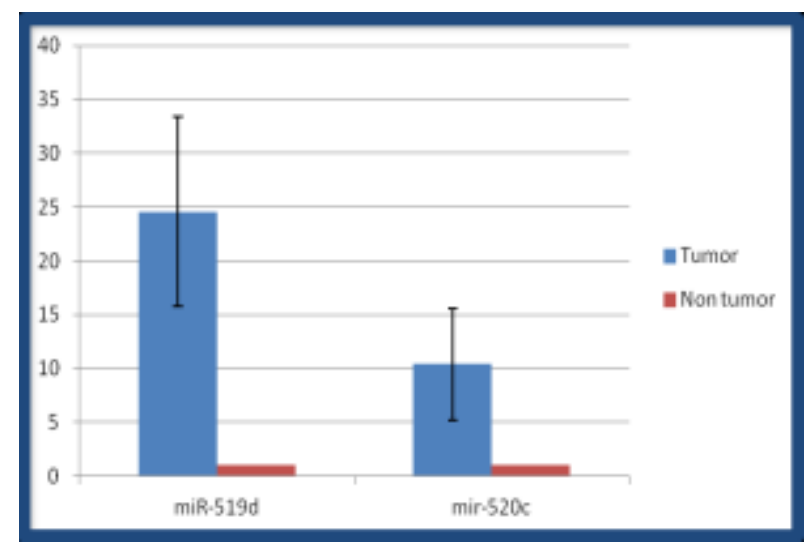

Fig. 1: Evaluation expression of miR-519d and miR-520c in esophageal tumor tissue compared with non-tumor adjacent tissue

Real-Time PCR demonstration figures of negative control sample and three miRNAs (miR-519d, miR-520c, and miR-16) as well as CT and Melting Curve graphs are shown in fig 2-4.

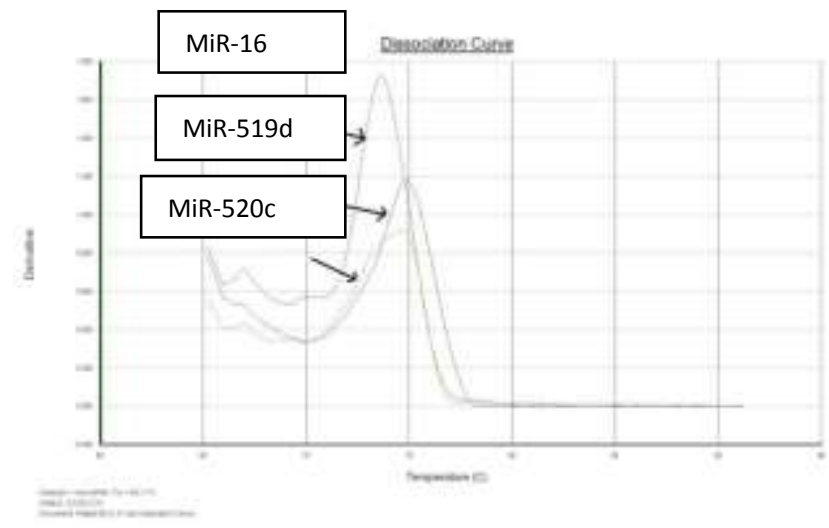

Fig. 2: melting curve graphs corresponding to the miR-16, miR-519d and miR-520c's genes expression

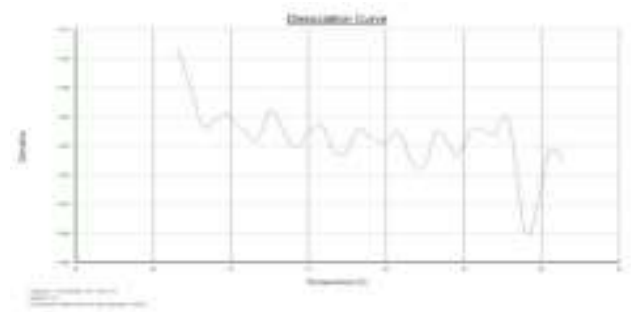

Fig. 3: melting curve graphs corresponding to negative control sam 


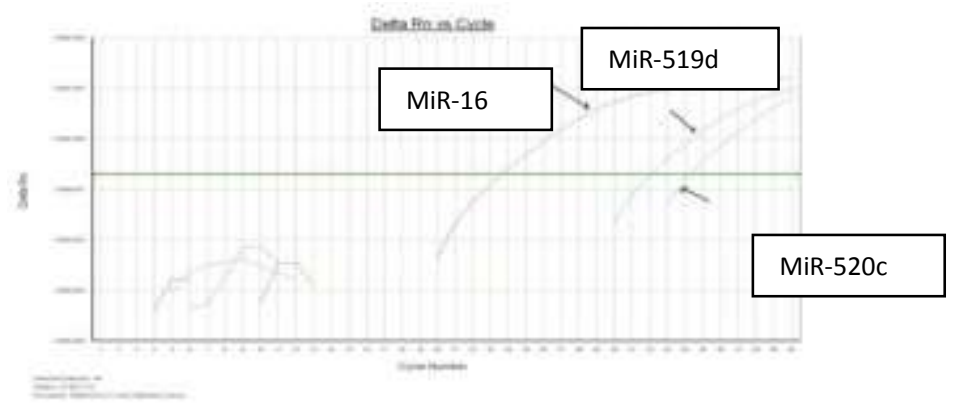

Fig . 4: Ct graph related miR-16, miR-519d and miR-520c's genes expression

Further analyses on patients' age and gender did not show any significant differences between males and females, and also between patients older and younger than 60 years old $(\mathrm{P}>0.05)$. Furthermore, further analysis on tumor differentiation (grade) did not show any significant differences between tumoral group tissues $(\mathrm{P}>0.05)$. The Pearson test was performed to investigate the correlation of miR-519d and miR-520c expression levels. The results signified a linear correlation between these two miRNAs with $\mathrm{R}=0.4$ and $\mathrm{P}$ value $<0.02$ (fig5).

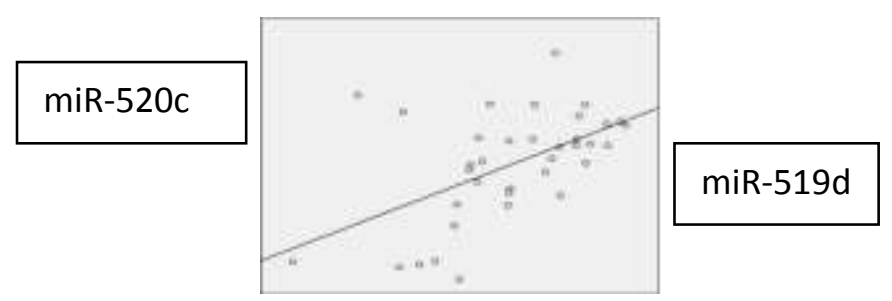

Fig. 5: linear correlation of miR-519d and miR-520c with $\mathrm{R}=0.4$ and $\mathrm{P}$ value $<0.02$. 
Moreover, for further evidential documents, Real-Time PCR's product's electrophoresis on 3\% agarose gel stained with cyber safe visualized miR-16, miR-519d and miR-520c's length by 62 , 64 and $65 \mathrm{bp}$, respectively.

\section{DISCUSSION}

EC is a poorly characterized deadly cancer with a 3-2 malignancy ratio among Iranian males and female, respectively $(1,26)$. Recent studies on molecular alterations associated with cancers have revealed the crucial roles of miRNA in cancer initiation and progression $(3,27)$. The present study provides evidence on sharp increases of 24.5 and 10.4 folds in the expressions of miR519d and miR-520c in ESCC's tumoral tissues compared to non-tumor marginal tissues which could indicates oncogenic roles of these two miRNAs in ESCC .Over expression of miR-519d and miR-520c has so far been reported in several cancers including breast, liver, prostate, fibroblast and hepatocellular carcinoma $(8,14-16,22-24,28-30)$. However, over expression of these two miRNAs has not been reported in ESCC yet.

According to former studies on miR-519d in hepatocellular carcinoma and prostate cancer, this miRNA performs its oncogenic roles by targeting P21, P53, AKT3 and TIMP2 $(8,15,17)$. Considering the oncogenic role of miR-519d in current study and further investigations based on certified database sources such as Pictar, mirWalk and Target scan and also reviewing the molecular mechanisms involved in the esophageal carcinogenesis process; P53, RAD23B, BID, P21, mKi67, PTEN and TIMP2 were identified as potential miR-519d's target gene in EC. Studies on P21 and Mki67 gene in EC point out their crucial role in cell cycle, prognosis, survival rate, cancer return and chemotherapy response in EC $(2,9,16,18,20,21,31-34)$. Overall, these studies raised the hypothesis that P21 and mKi67 could 
be main target genes of miR-519d in EC that still has not been investigated in this cancer. In addition, since samples collected in this experiment were from patients in Golestan Province, and also, due to the proven role of P53 in the pathogenesis of EC and extremely high mutation rate of P53 in ES patients in this area, this gene could well be considered as a target gene of miR-519d. To confirm this, however, further functional studies are needed . (35-37).

It has been previously reported that miR-520c has shown its oncogenic effects on stem cell, breast and prostate cancer by targeting P21 or CD44(22-24,38). It is worth adding that Correlation expression of these two miRNAs is able to indicate their oncogenic role through common carcinogenetic functional pathways like P21. Regarding the carried out investigations on miRNA target genes on previous reports which has shown that breast cancer in accompanied by increased risk of EC, it seems that CD44 can act as a target gene in EC as well, although more functional studies are needed to prove the hypothesis(6). So far, none of the genes mentioned above has been investigated as a miR-519d target gene in EC.

\section{CONCLUSION}

Suffice to say, to our knowledge, the present research is the first report on the spectacular increases in the expressions of miR-519d and miR-520c in ESCC, and supports the hypothesis of their oncogenic roles in ESCC. It also suggests them as potential targets for more research in clinical use and personalized treatment fields. 


\section{ACKNOWLEDGMENTS}

This project designed by Dr Seyed Javad Mowla. Nadia Ghasemian provided sample collection, bench work and writing the article. Clinical sample approval provided by Dr Alizadeh Sh. Dr Nader Mansour Samaei collaborated in advising and laboratory work. Masoumeh Ghasemi collaborated in sample collection and laboratory work. We would like to thank Dr Naser Behnampor for helpful comments on data analysis. This work was supported entirely by a research grant at Golestan University of Medical Science and the authors declare that they have no conflict of interest and all authors have approved the final article. 


\section{REFERENCES}

1. Sadjadi M, Marjani H, Semnani S, Nasseri-Moghaddam S. Esophageal Cancer in Iran: A Review. Middle East J Cancer. 2010;

2. Lin H, Cheng M F, Cheng M, Yu M, Lee S, Nieh S. p53, Ki67, and Histone deacetylase 4 Expression Associated with Clinicopathological Characteristics in Esophageal Squamous Cell Carcinoma. J Med Sci 2011; 31: 17-26.

3. Zhou S-L, Wang L-D. Circulating microRNAs: novel biomarkers for esophageal cancer. World J Gastroenterol WJG. 2010 May 21; 16: 2348-54.

4. Zhang C, Wang C, Chen X, Yang C, Li K, Wang J, et al. Expression profile of microRNAs in serum: a fingerprint for esophageal squamous cell carcinoma. Clin Chem 2010; 56: 1871-9.

5. Zhu L, Yan W, Rodriguez-Canales J, Rosenberg AM, Hu N, Goldstein AM, et al. MicroRNA analysis of microdissected normal squamous esophageal epithelium and tumor cells. Am J Cancer Res 2011; 1: 574-84.

6. Negrini M, Calin GA. Breast cancer metastasis: a microRNA story. Breast Cancer Res BCR 2008; 10: 203.

7. Marasa BS, Srikantan S, Martindale JL, Kim MM, Lee EK. MicroRNA profiling in human diploid fibroblasts uncovers miR-519 role in replicative senescence. Aging. 2010 2: $333-43$.

8. Hou Y-Y, Cao W-W, Li L, Li S-P, Liu T, Wan H-Y et al. MicroRNA-519d targets MKi67 and suppresses cell growth in the hepatocellular carcinoma cell line QGY-7703. Cancer Lett 2011; 307: 182-90. 
9. Wu S, Huang S, Ding J, Zhao Y, Liang L, Liu T, et al. Multiple microRNAs modulate p21Cip1/Waf1 expression by directly targeting its 3' untranslated region. Oncogene 2010; 29: 2302-8.

10. Busatto G, Shiao YH, Parenti AR, Baffa R, Ruol A, Plebani M, et al. p16/CDKN2 alterations and $\mathrm{pRb}$ expression in oesophageal squamous carcinoma. Mol Pathol 1998; 51: $80-4$.

11. Xing EP, Yang GY, Wang LD, Shi ST, Yang CS. Loss of heterozygosity of the Rb gene correlates with $\mathrm{pRb}$ protein expression and associates with $\mathrm{p} 53$ alteration in human esophageal cancer. Clin Cancer Res Off J Am Assoc Cancer Res 1999; 5:1231-40.

12. Lange CA, Yee D. Killing the second messenger: targeting loss of cell cycle control in endocrine-resistant breast cancer. Endocr Relat Cancer. 2011; 18: C19-C24.

13. Grady WM, Tewari M. The next thing in prognostic molecular markers: microRNA signatures of cancer. Gut. $2010 ; \mathbf{5 9 :} 706-8$.

14. Toffanin S, Hoshida Y, Lachenmayer A, Villanueva A, Cabellos L, Minguez B, et al. MicroRNA-based classification of hepatocellular carcinoma and oncogenic role of miR517a. Gastroenterol 2011; 140: 1618-28.e16.

15. Long Q, Johnson BA, Osunkoya AO, Lai Y-H, Zhou W, Abramovitz M, et al. Proteincoding and microRNA biomarkers of recurrence of prostate cancer following radical prostatectomy. Am J Pathol 2011; 179: 46-54.

16. Fornari F, Gramantieri L, Milazzo M, Palloca G. 209 Microrna-519d is up-regulated in hepatocellular carcinoma and sensitizes hec cells to doxorubicin treatment by targeting cdkn1a/p21. J Hepatol 54: 88. 
17. Fornari F, Milazzo M, Chieco P, Negrini M, Marasco E, Capranico G, et al. In hepatocellular carcinoma miR-519d is up-regulated by p53 and DNA hypomethylation and targets CDKN1A/p21, PTEN, AKT3 and TIMP2. J Pathol 2012; 227: 275-85.

18. Lin D-C, Shi Z-Z, Xue L-Y, Chen W, Xu X, Han Y-L et al. (Expression of cell cycle related proteins cyclin D1, p53 and p21WAF1/Cip1 in esophageal squamous cell carcinoma). Yi Chuan Hered Zhongguo Yi Chuan Xue Hui Bian Ji. 2010 May; 32: 45560.

19. Kurabayashi A, Furihata M, Matsumoto M, Ohtsuki Y, Sasaguri S, Ogoshi S. Expression of Bax and apoptosis-related proteins in human esophageal squamous cell carcinoma including dysplasia. Mod Pathol Off J United States Can Acad Pathol Inc 2001; 14: 7417.

20. Nakamura T, Hayashi K, Ota M, Ide H, Takasaki K, Mitsuhashi M. Expression of p21(Waf1/Cip1) predicts response and survival of esophageal cancer patients treated by chemoradiotherapy. Dis Esophagus Off J Int Soc Dis Esophagus ISDE. 2004; 17: 31521.

21. Shirakawa Y, Naomoto Y, Kimura M, Kawashima R, Yamatsuji T, Tamaki T et al. Topological analysis of p21WAF1/CIP1 expression in esophageal squamous dysplasia. Clin Cancer Res Off J Am Assoc Cancer Res 2000; 6: 541-50.

22. Iczkowski KA. Cell adhesion molecule CD44: its functional roles in prostate cancer. Am J Transl Res 2011; 3: 1-7.

23. Port M, Glaesener S, Ruf C, Riecke A, Bokemeyer C, Meineke V, et al. Micro-RNA expression in cisplatin resistant germ cell tumor cell lines. Mol Cancer 2011; 10: 52. 
24. Yang K, Handorean AM, Iczkowski KA. MicroRNAs 373 and 520c are downregulated in prostate cancer, suppress CD44 translation and enhance invasion of prostate cancer cells in vitro. Int J Clin Exp Pathol 2009; 2: 361-9.

25. 2- $\triangle \Delta \mathrm{CT}$ interpretation of gene copy number - PCR, RT-PCR and Real-Time PCR (Internet). (cited 2013 Aug 22). Available from: http://www.protocol-online.org/biologyforums-2/posts/7772.html

26. Enzinger PC, Mayer RJ. Esophageal Cancer. N Engl J Med 2003; 349: 2241-52.

27. Jansson MD, Lund AH. MicroRNA and cancer. Mol Oncol 2012; 6: 590-610.

28. Liu P, Wilson MJ. miR-520c and miR-373 upregulate MMP9 expression by targeting mTOR and SIRT1, and activate the Ras/Raf/MEK/Erk signaling pathway and NF-кB factor in human fibrosarcoma cells. J Cell Physiol 2012; 227: 867-76.

29. Rippe V, Dittberner L, Lorenz VN, Drieschner N, Nimzyk R, Sendt W et al. The Two Stem Cell MicroRNA Gene Clusters C19MC and miR-371-3 Are Activated by Specific Chromosomal Rearrangements in a Subgroup of Thyroid Adenomas. PLoS ONE. 2010 ; 5: e9485.

30. Patel N, Hoang D, Miller N, Ansaloni S, Huang Q, Rogers JT et al. MicroRNAs can regulate human APP levels. Mol Neurodegener 2008; 3: 10.

31. Yan W, Wistuba II, Emmert-Buck MR, Erickson HS. Squamous Cell Carcinoma Similarities and Differences among Anatomical Sites. Am J Cancer Res 2011; 1: 275300.

32. Ressiot E, Dahan L, Liprandi A, Giorgi R, Djourno X-B, Padovani L et al. Predictive factors of the response to chemoradiotherapy in esophageal cancer. Gastroentérologie Clin Biol 2008; 32: 567-77. 
33. Sherr CJ, Roberts JM. CDK inhibitors: positive and negative regulators of G1-phase progression. Genes Dev 1999; 13: 1501-12.

34. Liu J, Hu Y, Hu W, Xie X, Ela Bella A, Fu J et al. Expression and prognostic relevance of p21WAF1 in stage III esophageal squamous cell carcinoma. Dis Esophagus Off J Int Soc Dis Esophagus ISDE 2012; 25: 67-71.

35. Abedi-Ardekani B, Kamangar F, Sotoudeh M, Villar S, Islami F, Aghcheli K, et al. Extremely high Tp53 mutation load in esophageal squamous cell carcinoma in Golestan Province, Iran. PloS One 2011; 6: e29488.

36. Etemadi A, Abnet CC, Golozar A, Malekzadeh R, Dawsey SM. Modeling the risk of esophageal squamous cell carcinoma and squamous dysplasia in a high risk area in Iran. Arch Iran Med 2012; 15: 18-21.

37. Taghavi N, Biramijamal F, Sotoudeh M, Khademi H, Malekzadeh R, Moaven O et al. p16INK4a hypermethylation and p53, p16 and MDM2 protein expression in esophageal squamous cell carcinoma. BMC Cancer 2010; 10: 138.

38. Sheikh MS, Huang Y. Death receptor activation complexes: it takes two to activate TNF receptor 1. Cell Cycle Georget Tex. 2003 6: 550-2. 